\title{
¿Conflictos de intereses ocultos en publicaciones científicas en tiempos de COVID-19?
}

\author{
Hidden conflicts of interest in scientific publications in time of COVID-19?
}

\author{
Ivan D. Lozada-Martínez ${ }^{1,2 *}$, Luis R. Moscote-Salazar2,3 y Yelson A. Picón-Jaimes,2,4 \\ ${ }^{1}$ Centro de Investigaciones Médico-Quirúrgicas, Universidad de Cartagena, Cartagena, Colombia; ${ }^{2}$ Consejo Latinoamericano de Neurointensivismo \\ (CLaNi); ${ }^{3}$ Centro de Investigaciones Biomédicas, Universidad de Cartagena, Cartagena, Colombia; ${ }^{4}$ Centro Medico Medicien, Santiago de Chile, \\ Chile
}

Hemos leído con sumo interés el artículo publicado por Diéguez-Campa, et al. ${ }^{1}$, titulado The 2020 research pandemic: a bibliometric analysis of publications on COVID-19 and their scientific impact during the first months, en el que los autores hacen un excelente e innovador análisis bibliométrico sobre la publicación científica médica en los primeros meses de desarrollo de la pandemia de COVID-19.

Los autores revelan unos hallazgos interesantes sobre la distribución de las publicaciones científicas en revistas americanas, donde los artículos predominantes fueron "commentary", "editorial", "letter", "news" y "viewpoint" sobre temas de salud pública, con una tasa muy baja de artículos originales, a pesar de poseer una capacidad productiva en materia científica similar a la de China, país que acaparó la mayor proporción de estudios originales con el objetivo de indagar sobre el diagnóstico, la epidemiología, la fisiopatología, las poblaciones especiales y el tratamiento, en su mayoría. Pese a que en los Estados Unidos de América prevaleció el tipo de artículos cortos para esa época, el promedio de autores por artículo fue de 4.6, a diferencia de los procedentes de China, en los que fue de 6.5, pero claramente hay que señalar que los estudios llevados a cabo por investigadores chinos culminaron en artículos originales y revisiones narrativas o sistemáticas de la literatura, o metaanálisis, producto de experimentos en ciencias básicas y clínicas, los cuales justifican una autoría numerosa ${ }^{1}$. Contrario a lo observado en los Estados Unidos de América, donde se evidenció que fue el segundo país con mayor número de autores sobre COVID-19, y el país donde se publicaron la gran mayoría de los manuscritos ligados al mismo tópico. Teniendo en cuenta el número de autores americanos en promedio por artículo publicado en revistas americanas, y que alrededor del $60 \%$ de estos artículos corresponden a "commentary", "editorial", "Ietter", "news" y "viewpoint"1, vale la pena plantear el siguiente interrogante: ¿existen conflictos de intereses ocultos en la publicación científica sobre COVID-19 en los autores y las revistas americanos? Los resultados sugieren que sí, y de ser esto cierto, constituye una falta ética grave.

El Comité Internacional de Editores de Revistas Médicas establece que el proceso científico y la credibilidad de los artículos publicados son una responsabilidad compartida, la cual depende de la transparencia con que se manejan los conflictos de intereses durante la planificación, la implementación, la escritura, la revisión por pares, la edición y la publicación ${ }^{2}$. Así mismo, declaran que la sospecha de que existe conflicto de intereses es tan importante como su existencia real ${ }^{2}$. Y aunque los conflictos de tipo financiero son los que con mayor frecuencia ponen en duda la credibilidad de los resultados de un estudio, la coautoría por cortesía, competencia académica, influencias personales o rivalidades, entre

\section{Correspondencia:}

*Ivan D. Lozada-Martínez

E-mail: ivandavidloma@gmail.com
Disponible en internet: 30-09-2021 Arch Cardiol Mex. (ahead of print) www.archivoscardiologia.com
Fecha de recepción: 11-11-2020

Fecha de aceptación: 20-08-2021 :20000510

CC BY-NC-ND (http://creativecommons.org/licenses/by-nc-nd/4.0/). 
otras razones, ocasiona inseguridad en la formalidad y el rigor de la ciencia, sobre todo de la ciencia médica, la cual se ha visto fuertemente criticada en estos tiempos por la población en general ${ }^{3}$. Estas faltas, más que generar solo un ambiente de crítica entre la comunidad, los actores de la salud, los investigadores y las instituciones de salud, originan desconfianza en la evidencia médica, con lo que disminuye la adherencia a las recomendaciones en materia de promoción de la salud y prevención de la enfermedad, así como a los tratamientos farmacológicos, y peor aún, da pie a que se intensifiquen las practicas pseudocientíficas ${ }^{4}$.

En aras de proteger la ciencia, asegurar su calidad y facilitar el ejercicio de conductas basadas en la evidencia, es necesario, ante todo, consolidar la ética de los autores, editores y demás participantes de las revistas científicas en general. La sospecha de la existencia de conflictos de intereses que distorsionen la veracidad de una publicación científica debe ser denunciada de manera prudente, rápida y respetuosa, mediante cartas al editor en las que se expongan las fallas e implicaciones a corto, mediano o largo plazo de los resultados o del proceder de los autores.

\section{Financiamiento}

La presente investigación no ha recibido ninguna beca específica de agencias de los sectores público, comercial o sin ánimo de lucro.

\section{Conflicto de intereses}

Ninguno.

\section{Responsabilidades éticas}

Protección de personas y animales. Los autores declaran que para esta investigación no se han realizado experimentos en seres humanos ni en animales.

Confidencialidad de los datos. Los autores declaran que han seguido los protocolos de su centro de trabajo sobre la publicación de datos de pacientes.

Derecho a la privacidad y consentimiento informado. Los autores declaran que en este artículo no aparecen datos de pacientes.

\section{Bibliografía}

1. Diéguez-Campa CE, Pérez-Neri I, Reyes-Terán G, Flores-Apodaca IA, Castillo-Ledón-Pretelini J, Mercado-Bautista O, et al. The 2020 research pandemic: a bibliometric analysis of publications on COVID-19 and their scientific impact during the first months. Arch Cardiol Mex. 2020 Nov 4. doi: 10.24875/ACM.20000370. Online ahead of print.

2. International Committee of Medical Journal Editors. Disclosure of financial and non-financial relationships and activities, and conflicts of interest. (Consultado el 10 de noviembre de 2020.) Disponible en: http://www.icmje.org/recommendations/browse/roles-and-responsibilities/author-responsibilities--conflicts-of-interest.html

3. Yang KC, Torres-Lugo C, Menczer F. Prevalence of low-credibility information on twitter during the COVID-19 outbreak. arXiv. 2020; arXiv:2004.14484

4. Mainous A. A towering Babel of risk information in the COVID-19 pandemic: trust and credibility in risk perception and positive public health behaviors. Fam Med. 2020;52:317-9. 